\title{
Mapping in Monochrome: Finalists from MonoCarto 2019
}

\section{INLAND NORTHWEST WASHINGTON ART DECO MAP, BY SARAH BELL}

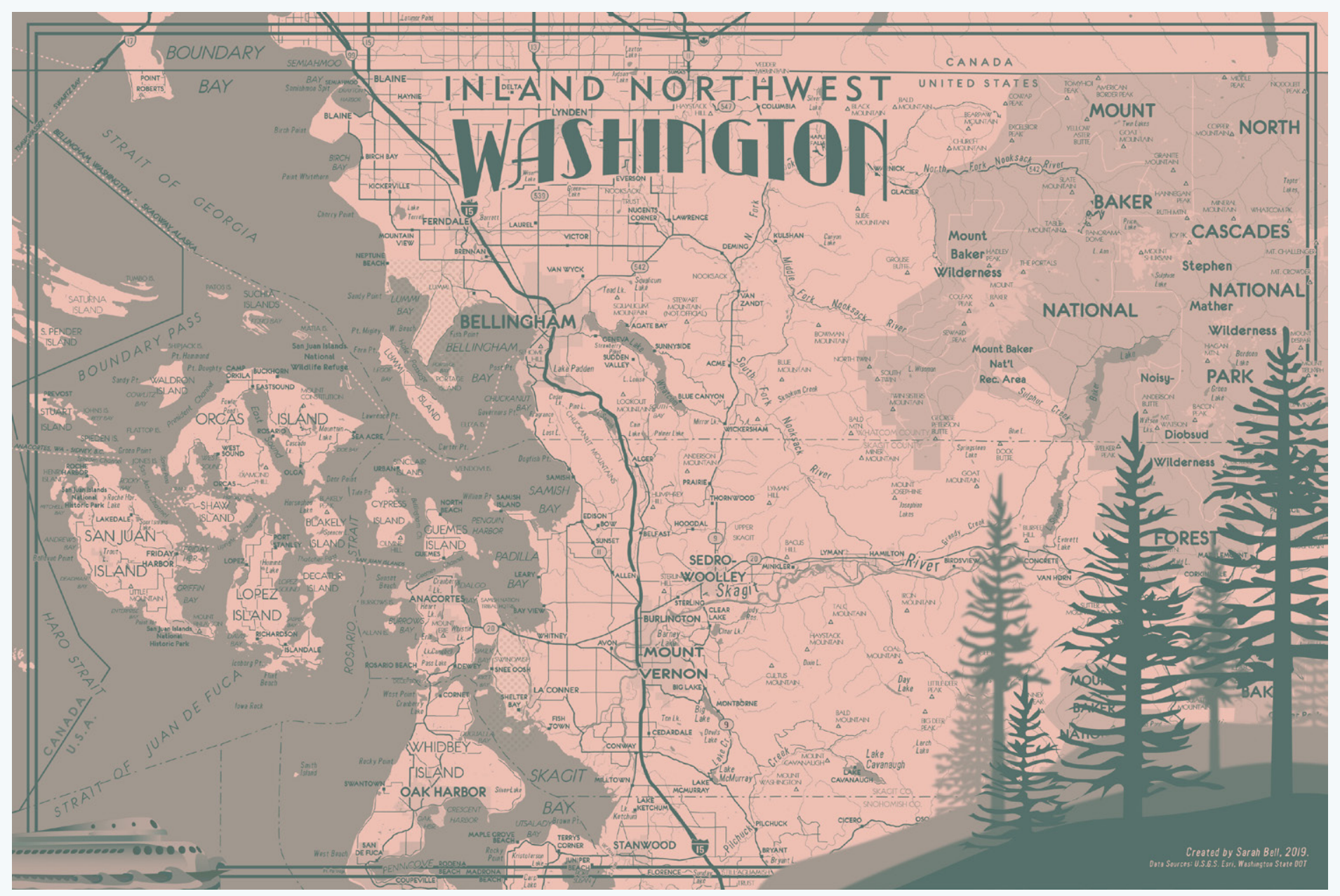

"Even within the constraints of a single color it manages to deliver a lot of information in a clear way without seeming too cluttered." Hans van der Maarel, MonoCarto 2019 competition judge

In 2019, Daniel P. Huffman organized MonoCarto, a map design competition with one primary stipulation: participants could only employ one foreground color, one background color, and any mixtures in-between. This initial foray was an unqualified success; over 150 cartographers from around the world submitted maps to be judged by a panel carefully selected by Huffman to represent the range of perspectives in today's field. This edition of Visual Fields exhibits selected finalists, and coincides with an in-person exbibition at the 2019 NACIS Annual Meeting in Tacoma, Washington. The author of this piece, Visual Fields section editor Jake Coolidge, also served on the judging panel. 
For MANy CARTOGRAPHERs, a monochrome design challenge arises from limitations imposed by the medium-the client needs a map for a book she's writing, and the publisher's budget only allows for printing with black ink; or, maybe a map will be circulated in classrooms and must still convey its message after it's been reproduced on a photocopier. The examples from recent history are varied, but could be typically boiled down to a common bottom line: full-color lithography is expensive, and printing in one color provides significant reductions in cost.

Now that most maps are designed for screens first and foremost, and distributed online, we can reconsider the role, the affordances, the power of monochrome maps in a digital, color-saturated medium. In a talk given at the NACIS meeting in Norfolk in 2018 (youtu.be/ptKDS1Z8Oro), Huffman expressed dissatisfaction with the arbitrary use of color. With so many colors at the cartographer's disposal, the thoughtful use of other visual variables, including texture, saturation, and value, is often lacking. Huffman implored cartographers to restore intentionality to the use of colors in maps, and in doing so, restore colors' power as cartographic symbols.

A great monochrome map will not leave the viewer wanting for more color-instead, with that one selected color, the cartographer can express specific aspects of the depicted geography with great acuity. It is a special sort of cartographic challenge that can spark a designer's creativity in new, unanticipated ways. And as Huffman noted, monochrome maps are innately more accessible, as they convey the same amount of information to viewers with color vision impairments and those who perceive the full spectrum.

It might be worth noting that monochrome is not strictly black on white. Returning to its print antecedent, monochrome can be black ink, printed at varying densities, on white paper, but it can also be blue ink on cream-colored paper, or reddish-brown ink on a manila folder. Black-onwhite is well represented in these selected maps, but we also see white-on-black, as well as blue-, red-, or greenon-white, and in the case of Sarah Bell's striking Inland Northwest Washington Art Deco Map above, the design simulates green ink printed on pink-tinted paper. Huffman was clear in permitting the use of simulated paper colors at the contest's beginning.

The finalists displayed here embrace the limitations imposed by monochrome, and in doing so, provide fine examples of creativity in layout design, typography, and map symbolization. Explore the entries on the following pages and find inspiration for your next monochrome mapping project. Accompanying each entry is a quote from the MonoCarto 2019 panel of judges; to read the complete commentaries, visit Huffman's blog: somethingaboutmaps.wordpress.com/monocarto-2019-winners.

\section{THE TOPOGRAPHY OF WEALTH IN LA, BY NICK UNDERWOOD}
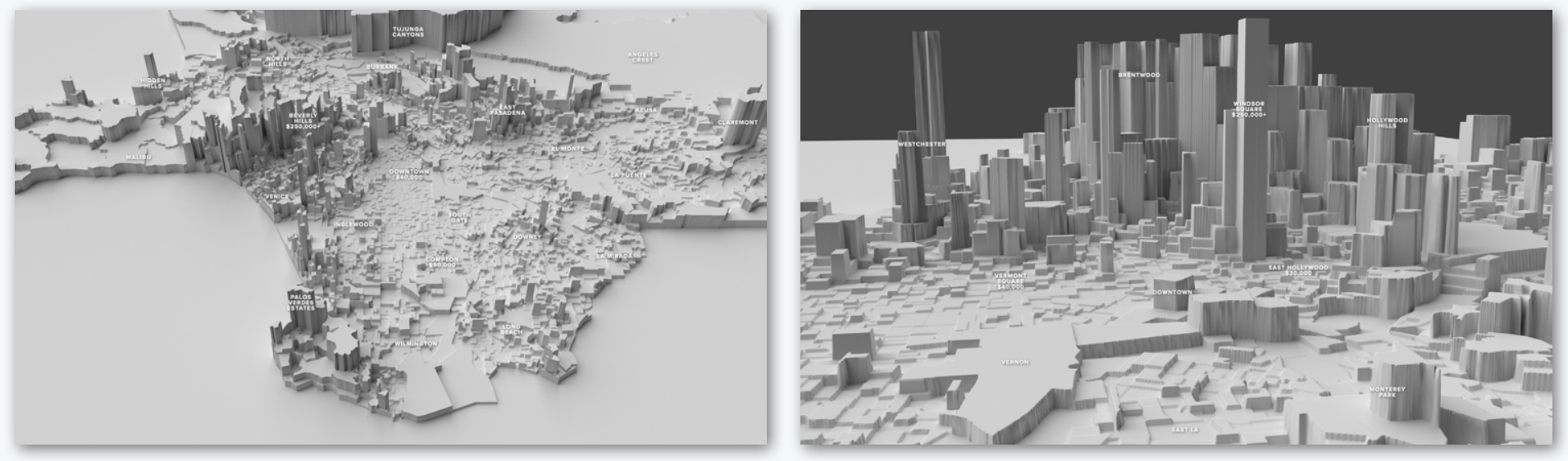

Shown are stills from an interactive visualization, which can be viewed here: nunderwood6.github.io/topography_of_wealth.

"The minimal presentation (note the stark grey sky in the background) offers a sort of early CGI feeling. But I'm not sure it needs more than that; color might simply muddy the clarity of the shapes. Some light and shadow is all that's needed to see what's going on." - Daniel Huffman 


\section{THE SUM OF ITS PARTS, BY PETE KING}

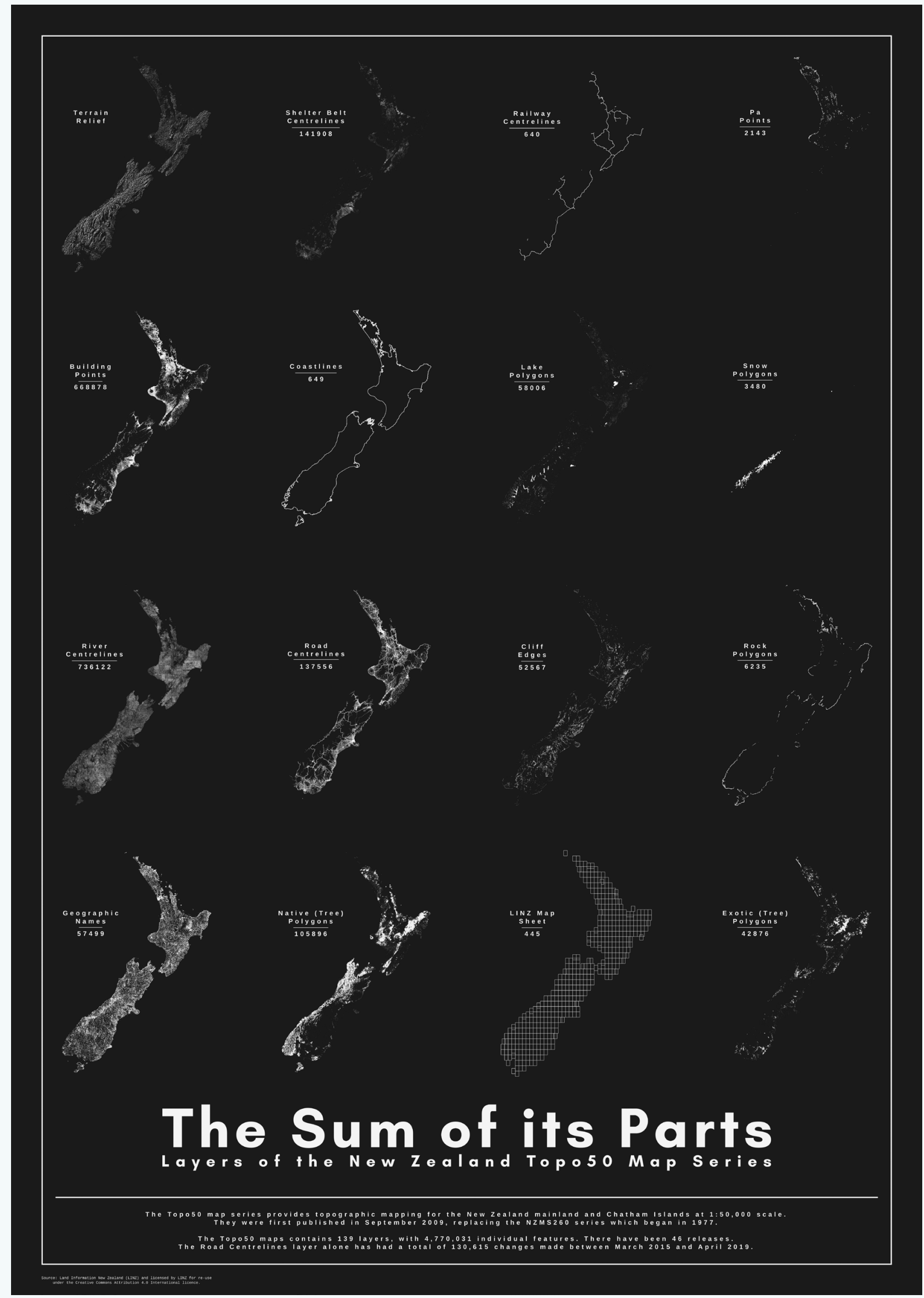

"The simple and straight-forward design of "The Sum of its Parts" is a map where the content drives the palette, rather than where the palette drives the content." - Ginny Mason 
APPALACHIAN TRAIL FOLD OUT SHELTER REFERENCE \& JOURNALATOP ADVENTURE MAP, BY JOHN NELSON AND ERICH RAINVILLE -

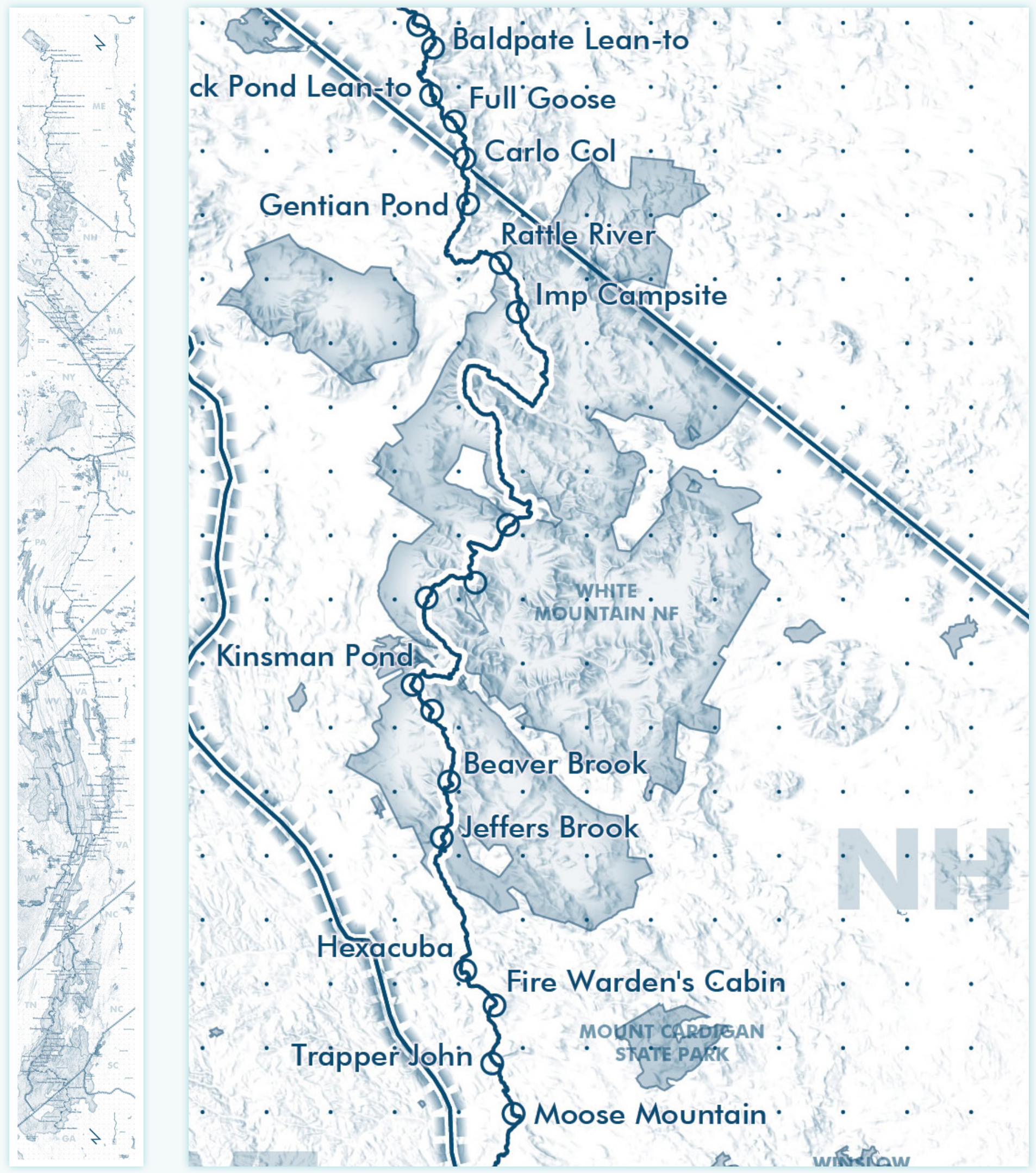

"The fact that it is monochrome seems entirely appropriate as we are missing no information that is relevant to the imagined journey; and no doubt its monochrome-ness adds to its beauty." - Arzu Çöltekin 

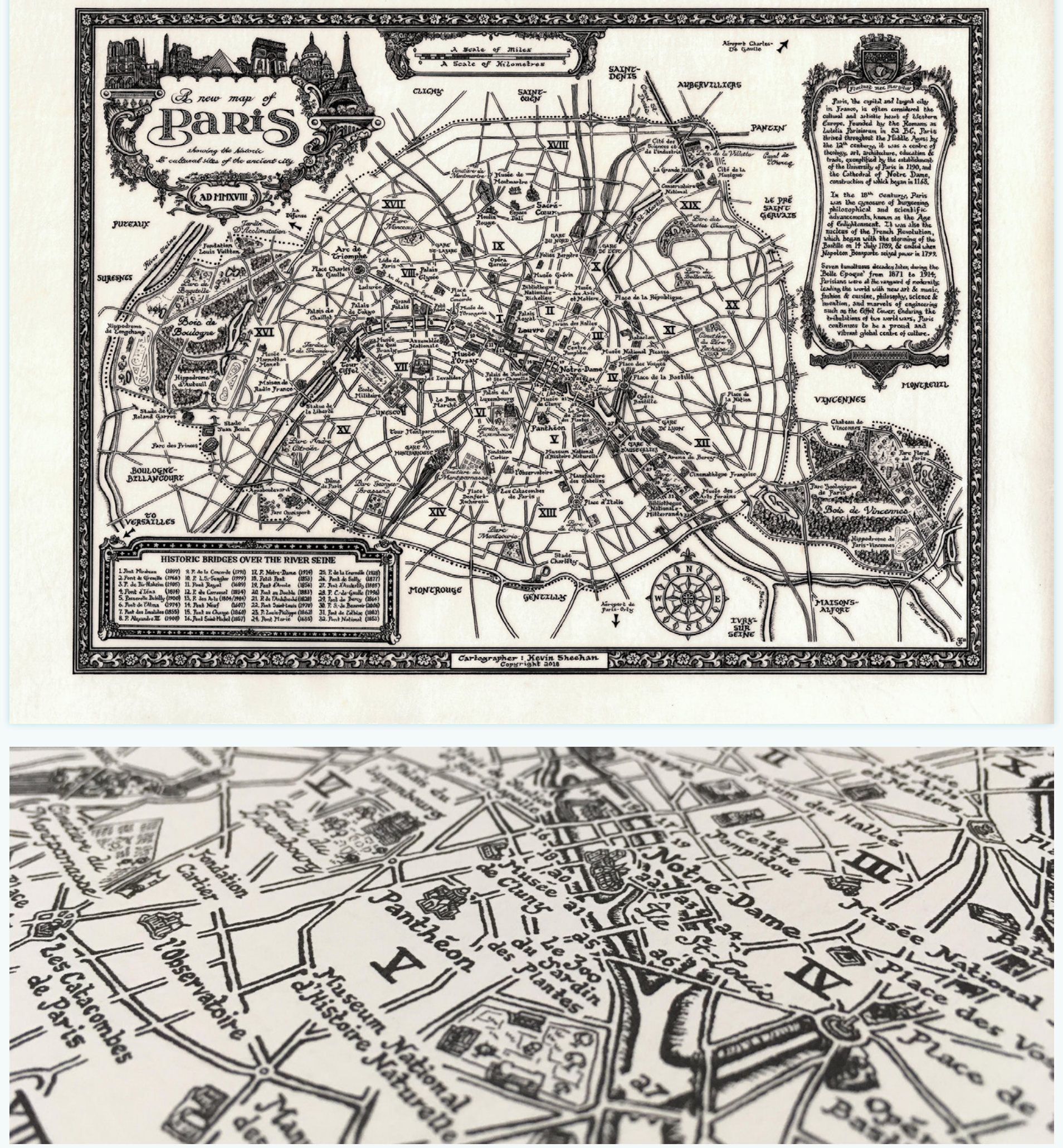

"Purely monochrome in ink and calf vellum, this stunning illustration of Paris displays true craftsmanship." —Sarah Bell 


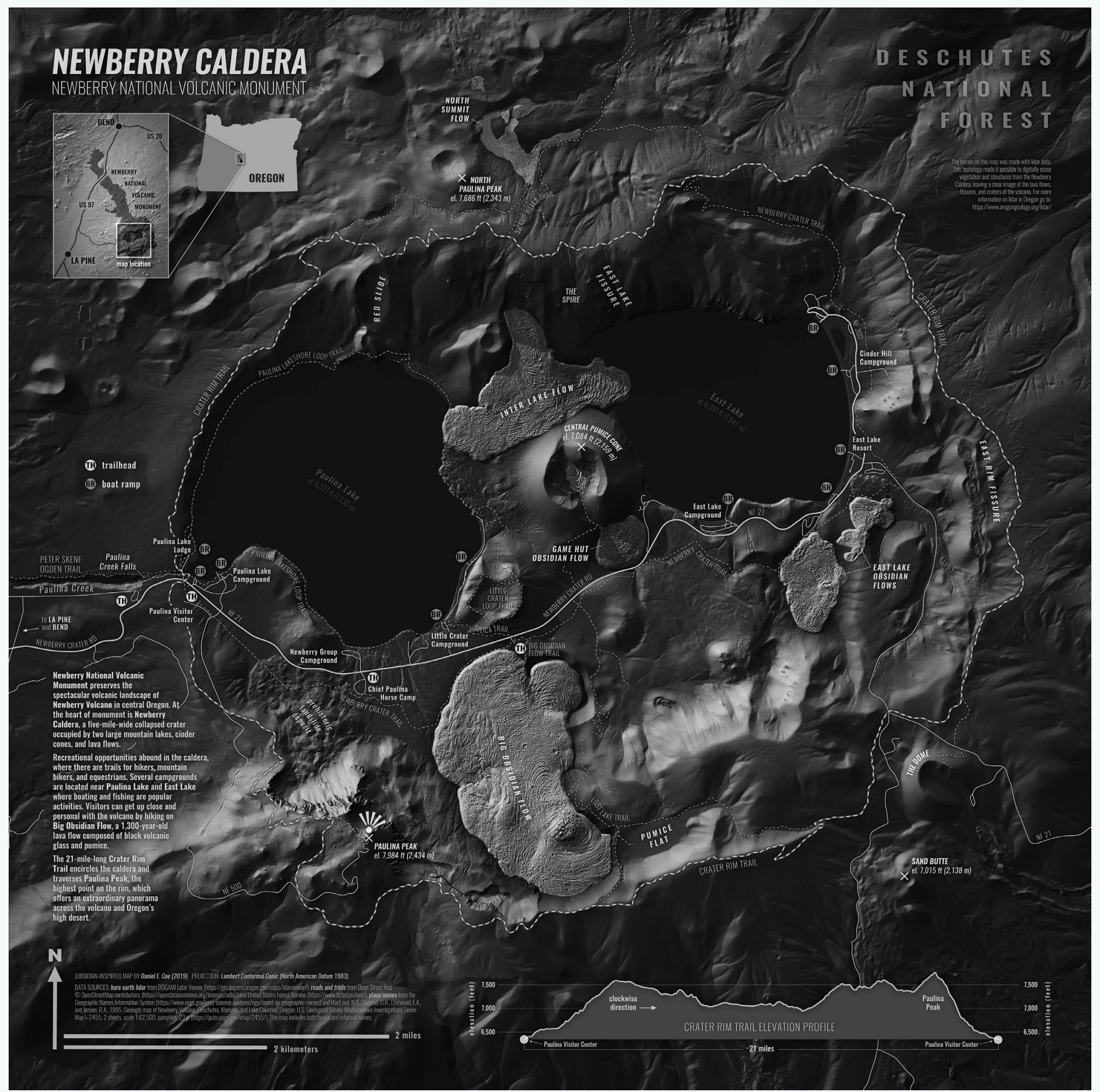

"The author has shown monochrome is not always a limitation in cartography, but at times an asset that can enhance the relationship between reader and nature, making this one of the best examples of contemporary monochrome maps." - Brooke Marston 
FLOW MAP OF THE GRAND RIVER, BY WARREN DAVISON

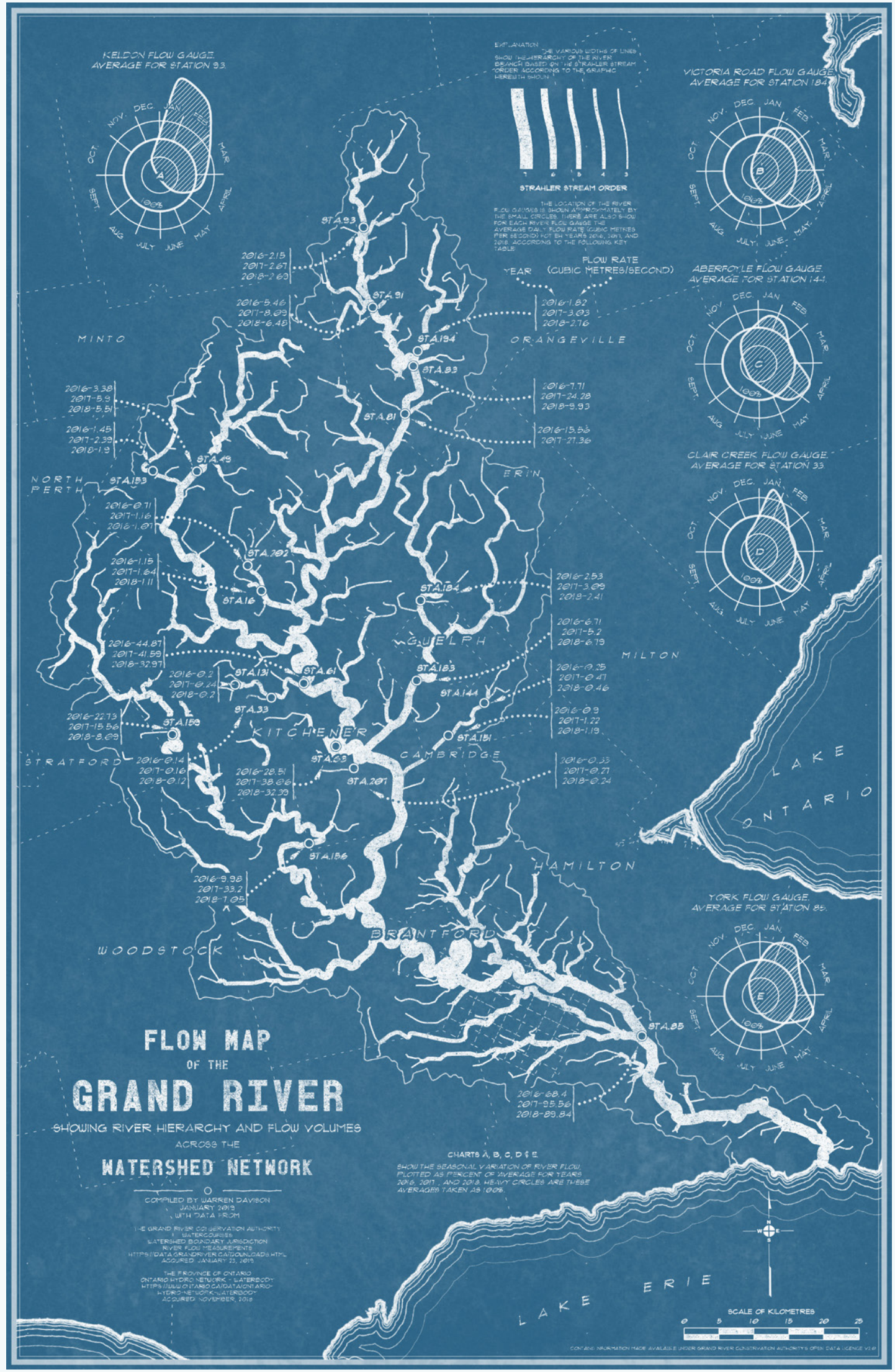

"By primarily using lines (of various thicknesses and dot/dash patterns) as the central graphic system, the map can remain monochrome while offering optimal readability. It is a very beautiful map." -Xemartin Laborde 


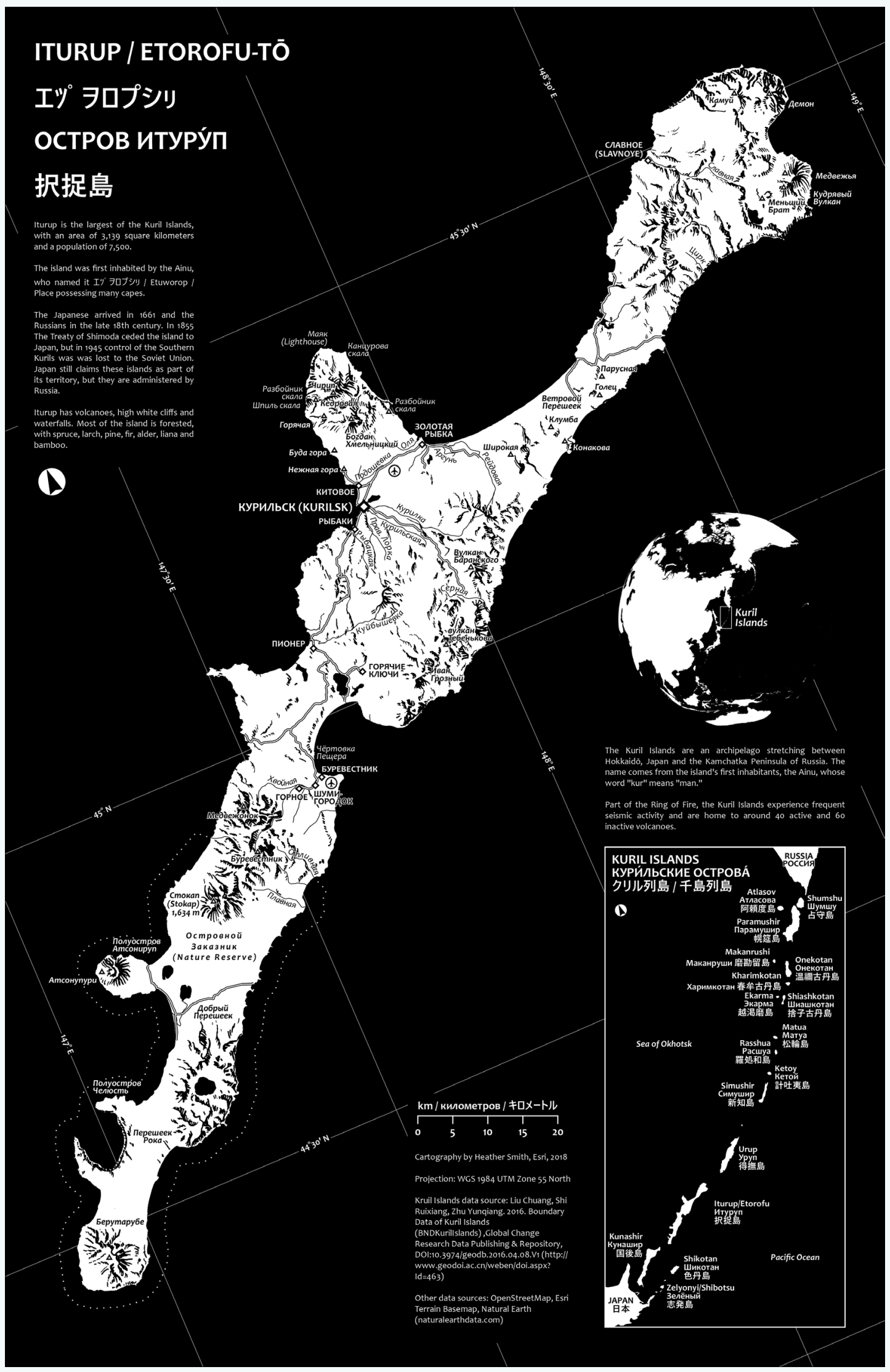

"The stark black and white contrast provides a fantastic figure-ground relationship, while careful attention to line weights really makes the various map features stand out." -Martha Bostwick 


\section{MONOCHROME DRIVE TIME MOUNTAINS, BY CRAIG TAYLOR, ITO WORLD}
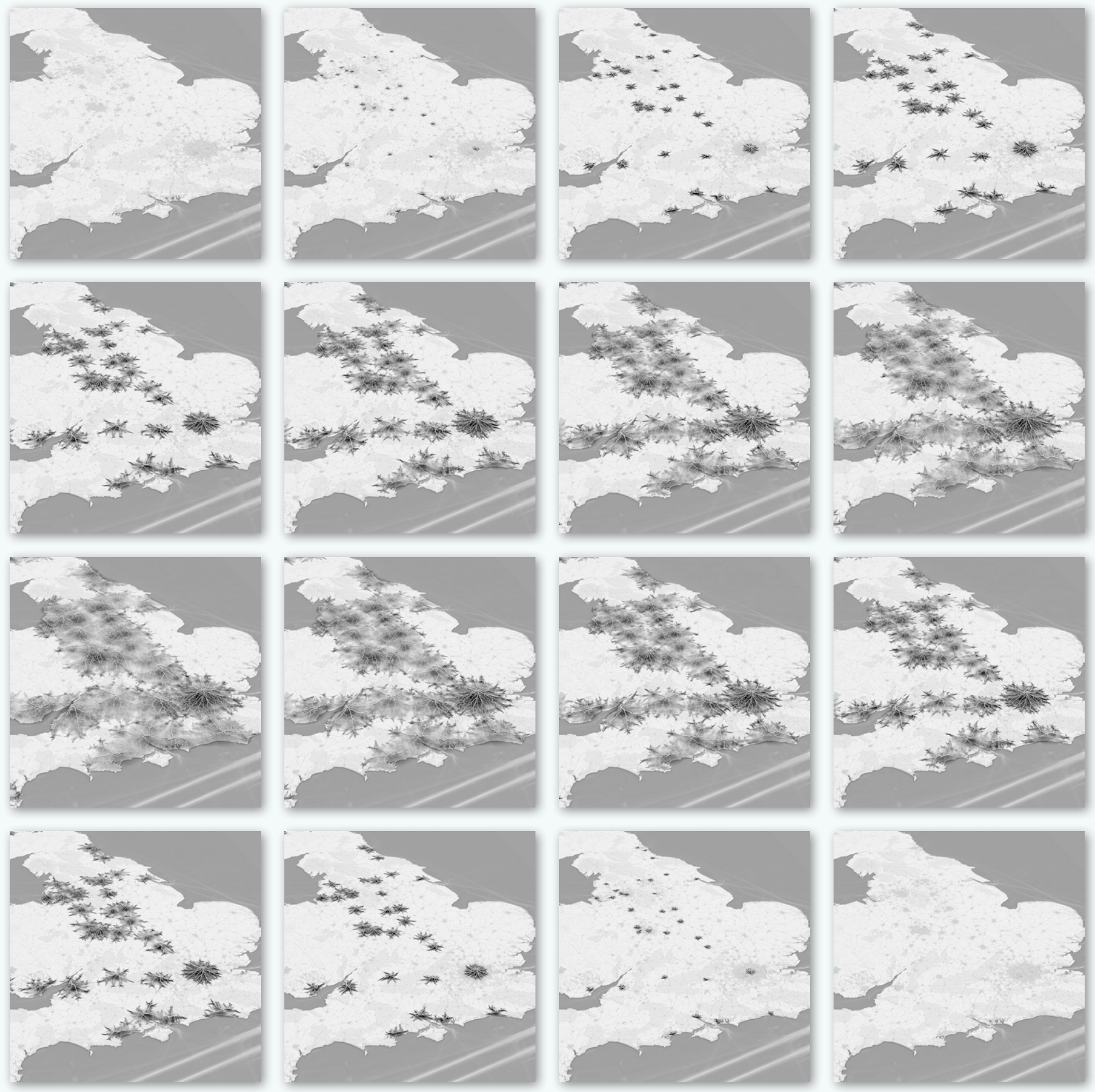

Shown are stills from an animated visualization, which can be viewed here: www.dropbox.com/s/4q4dnz6jonnv7oh/BD.mp4? $d l=0$.

"Craig's design decision to size the roads according to their width and likely traffic volumes produces a map whose appearance also references the small blood vessels that feed into larger ones to bring life to the body. The balance of greyscale variations provides enough contrast to clearly delineate the growth and diminishment of the mountains along the road networks and focuses the reader's attention within the changing scene. Finally, the measured pace of the animation contributes to the sense of a living, breathing commute." - Amy Griffin 

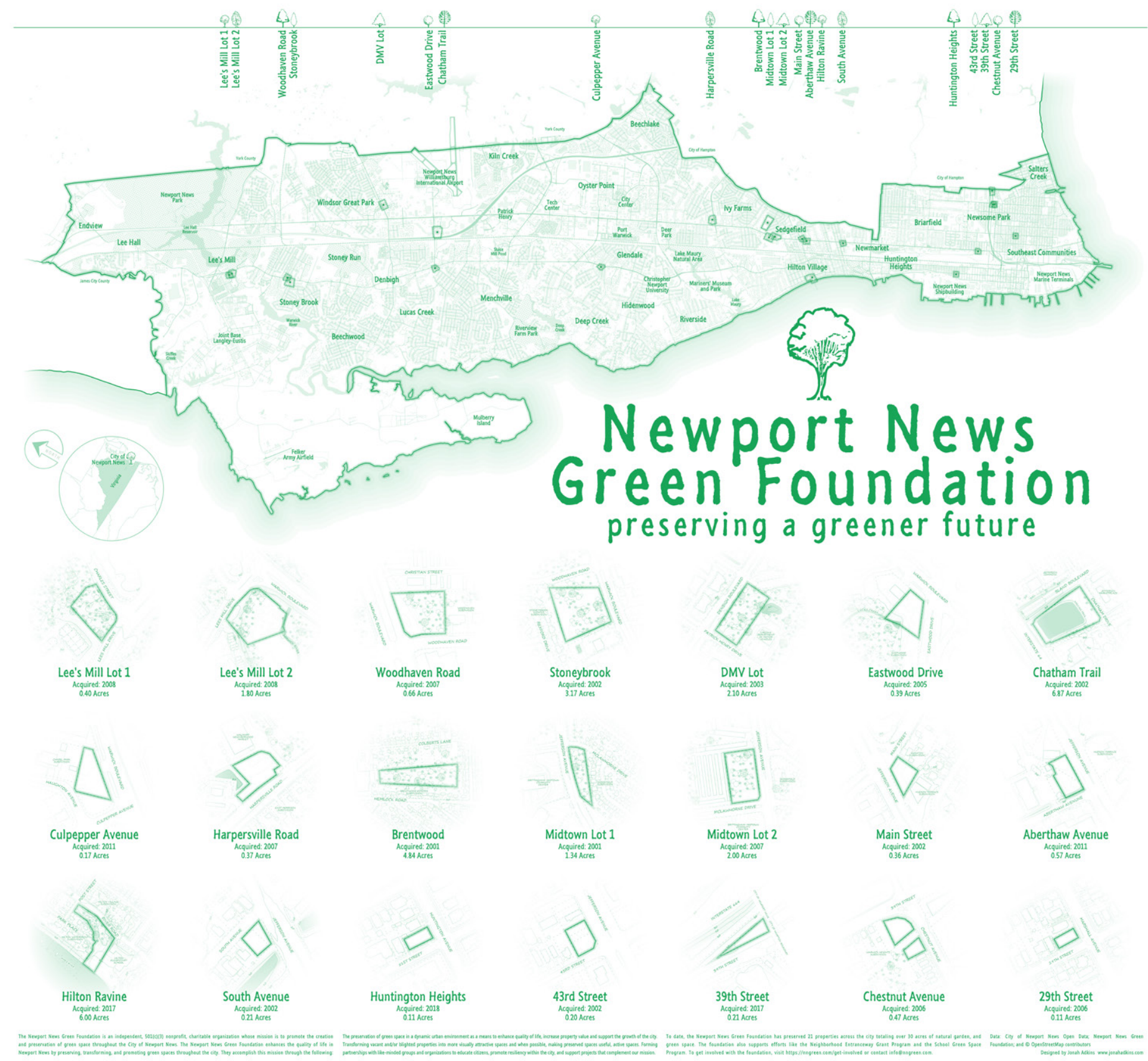

"It is a good map of the achievements of a local non-profit. The general conception and layout are elegant and understated, and the map should be inexpensive to print." - Mark Denil 


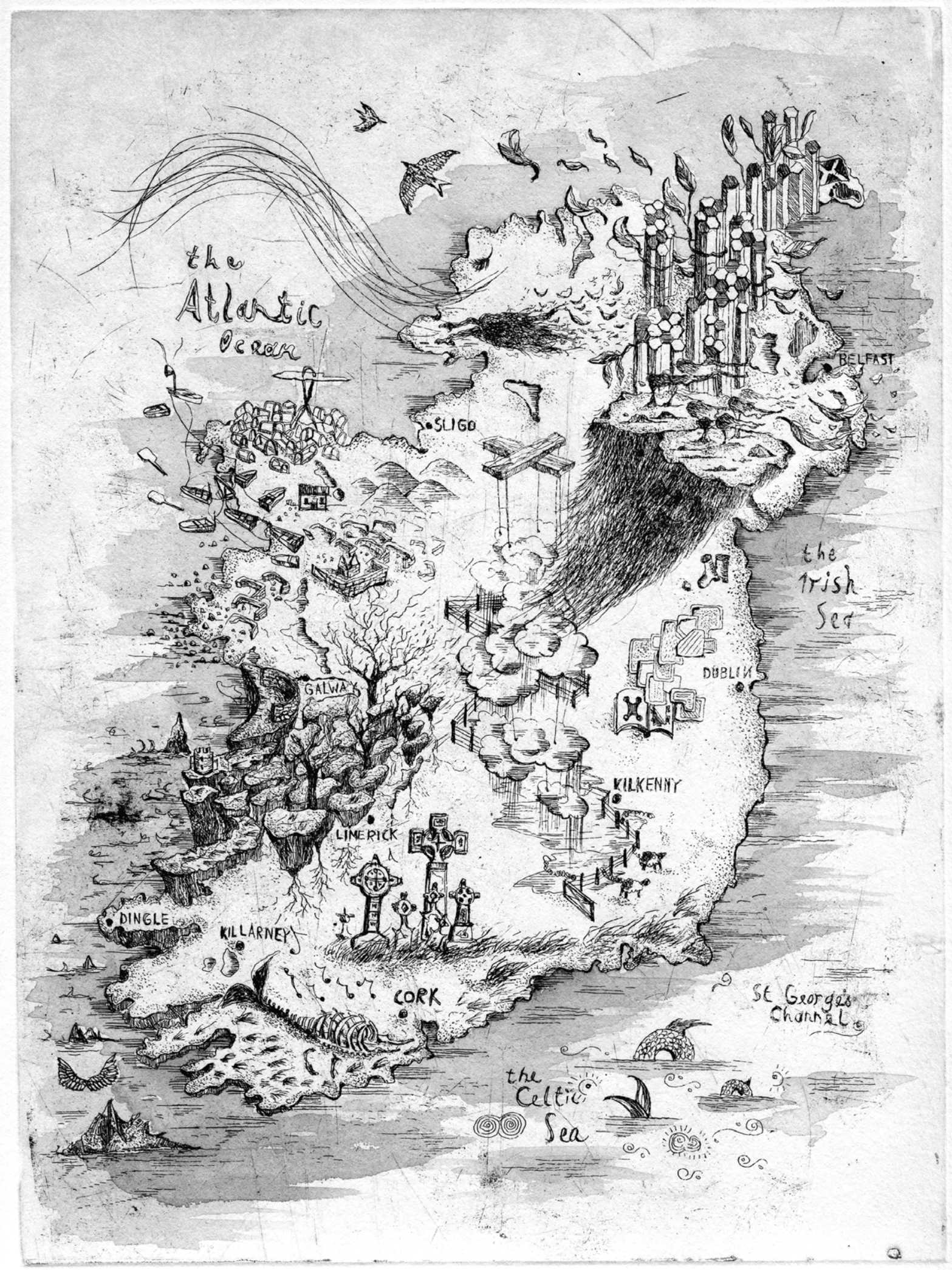

"The mixture of a solid fill to frame the coastline, and the variation in density of textures and patterns helped differentiate complex illustrations from each other, making this a detailed but easy-to-absorb map in monochrome. I kept getting lost in this map in the best way possible, noticing the intricate illustrated details of symbols and physical features. And that coastline. . I notice new details every time I look at it." - Lauren Tierney 


\section{THE LOST TREASURES OF LA ISLA DEL COCO, BY JAKOB LISTABARTH, UNDER THE SUPERVISION OF MANUELA SCHMIDT}

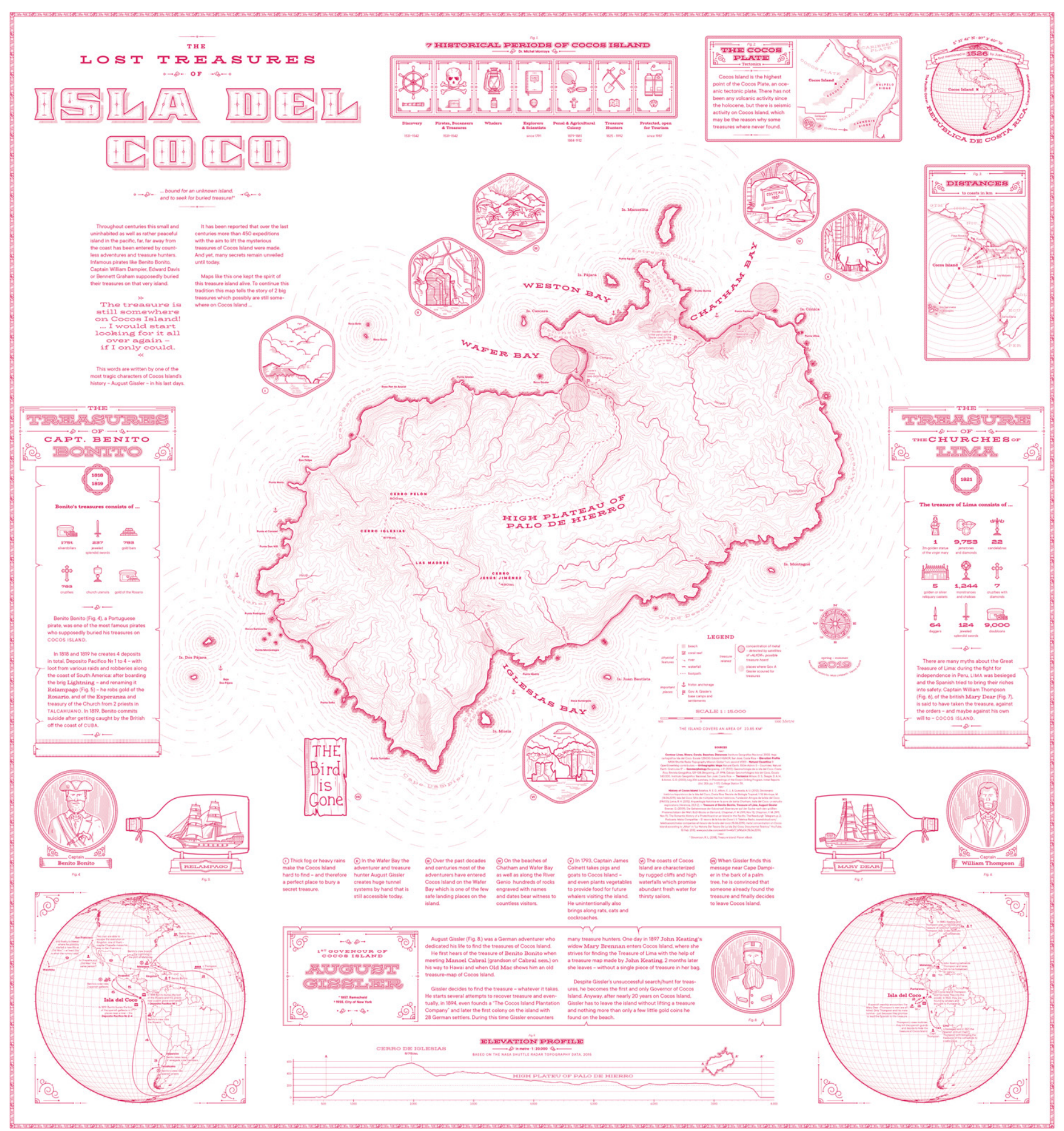

"Continuous monochrome is tough enough, but Jakob Listabarth takes the challenge even further and uses this map's sole ink at 100\% strength only. He is only able to distinguish feature types from each other using line weight, dot/dash patterns, and hachure shading. This he does excellently, and I continue to be impressed by how much information is shown, and how clearly each layer is distinguished from the others when they are all, after all, exactly the same version of magenta." -Daniel Huffman 


\section{PERU: A RELIEF MAP, BY SHANNON GONZALES}

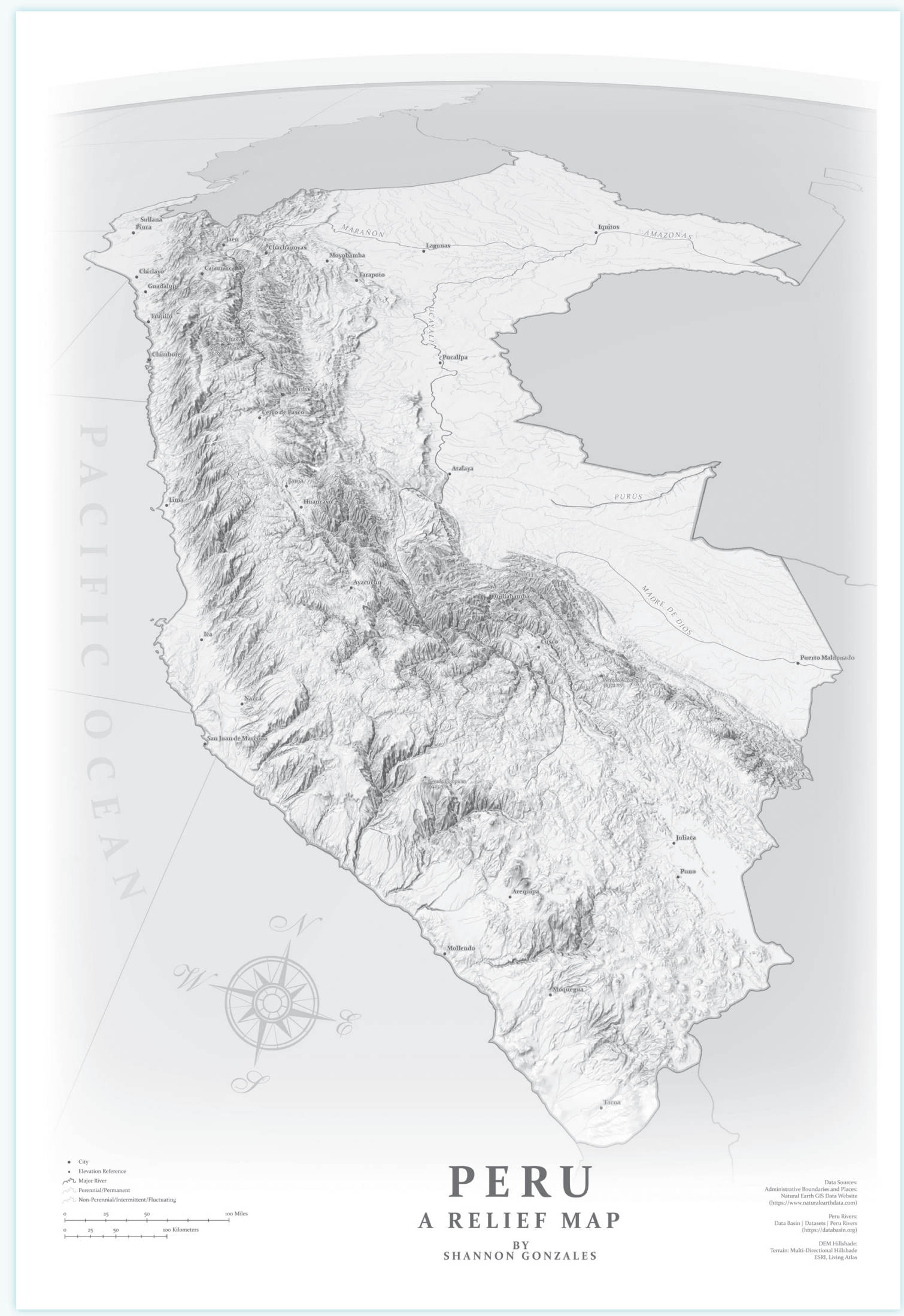

"This map pleased me because of its perspective projection. This view brings realism, makes the exaggerated relief appear more 3D, and offers a horizon that gives the impression that we are looking at Peru from space. The use of this map projection reminds me of Richard Edes Harrison's impressive orthographic maps" -Xemartin Laborde 


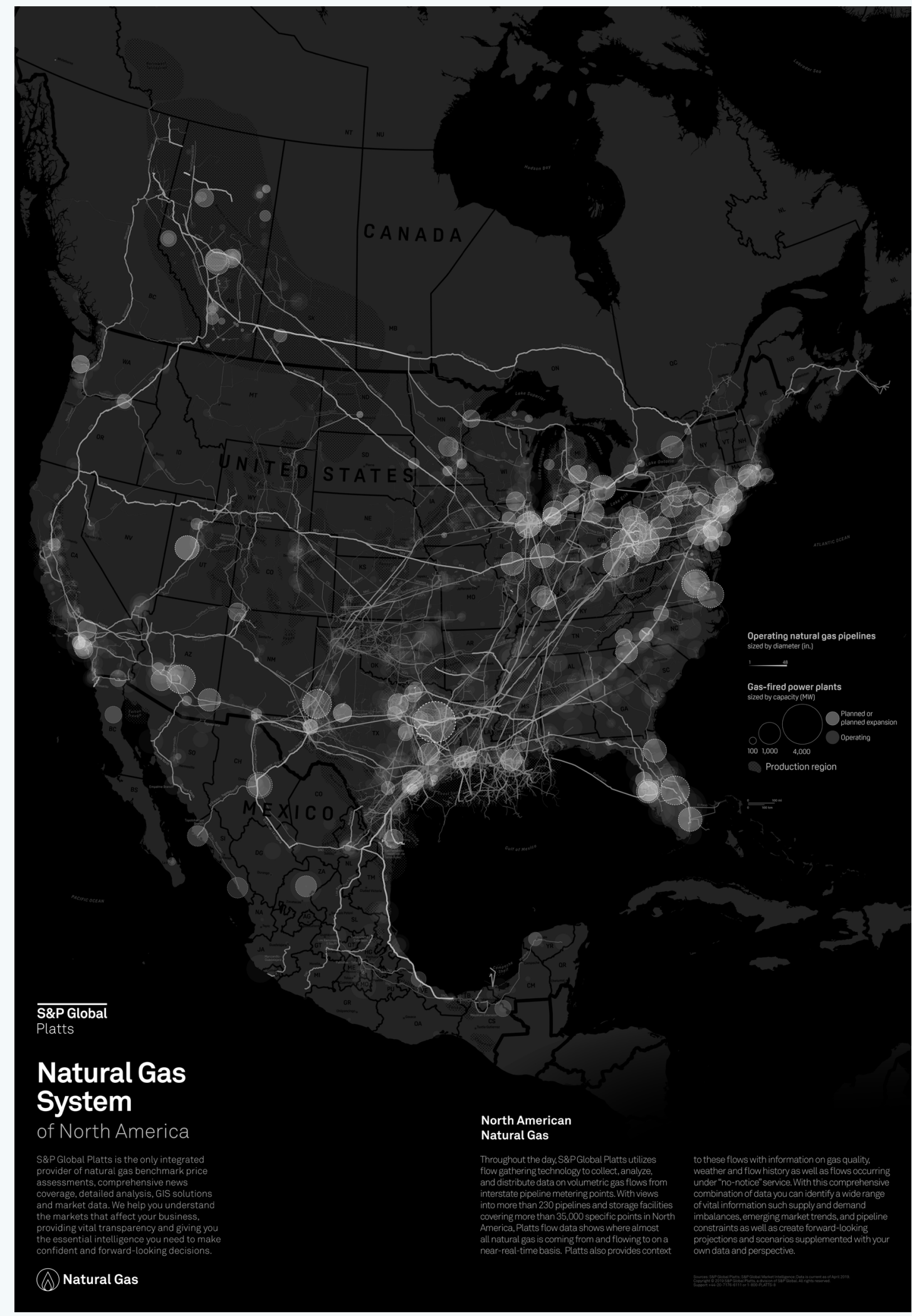

"... we're used to seeing such maps with loads of color as the cartographer tries what they can to discern one element from another. In this map the margins of error are tiny, slightly more or less transparency on the circles would have made them indistinguishable, a notch up or down on the gradient applied to the pipelines would have resulted in a messy tangle of lines shining through. ... My favourite maps are those that appear effortless to all but the most specialist readers who can appreciate the many, many tiny tweaks required to create them. In that regard this map really delivers." -James Cheshire 